\title{
Actuator and Sensor Selection for Robust Control of Aeroservoelastic Systems
}

\author{
Claudia P. Moreno, Harald Pfifer and Gary J. Balas ${ }^{1}$
}

\begin{abstract}
This paper proposes an approach for actuator and sensor selection for a small flexible aircraft. The approach is based on the synthesis of robust controllers accounting for model uncertainty. The objective is to find, out of a finite set of actuator/sensor configurations available in the aircraft, the best configuration that provides sufficient robustness and desired performance. The results show that the ability to stabilize and achieve performance objectives of aeroservoelastic systems is highly dependent on the selection of actuators and sensors for feedback control.
\end{abstract}

\section{INTRODUCTION}

The need for improved performance and reduced operational costs has led modern aircraft designers to adopt lightweight, flexible wings. A technical challenge associated with these designs is that the large deformations in flight of the wings lead to an adverse interaction between the aircraft aerodynamic forces and structural forces. These adverse interactions produce excessive vibrations that can degrade flying qualities and may result in severe structural damages or catastrophic failure.

Several control strategies have been proposed to address the excessive vibrations in flexible aircraft [1]-[4]. However, research in this field remains active as it is difficult to develop controllers that are insensitive to model errors yet still achieve excellent flying qualities. Moreover, selection of adequate sensor measurements and control effectors plays a critical role in the control design process. A wrong choice of actuator and sensors may put fundamental limitations on the performance and robustness that cannot be overcome by available advanced control design techniques. Hence, special attention to selection of input and output measurements for feedback control is required as an initial step in the design of controllers in order to successfully meet the desired performance.

Several methods to address the actuator and sensor selection problem are found in the literature. A majority of these methods can be grouped as control design independent or dependent methods. Control design independent methods are based on measures of controllability and observability of the plant. These include the frequency dependent condition number of the plant and the relative gain array (RGA) measure [5]. These measures were introduced initially to assess the robustness and interactions in decentralized control.

Control design dependent methods require a complete controller design to decide on a set of actuator and sensor

\footnotetext{
${ }^{1}$ Claudia P. Moreno, Harald Pfifer and Gary J. Balas (deceased 2014) are with the Department of Aerospace Engineering and Mechanics, University of Minnesota, Minneapolis, Minnesota, 55455 moren 148 @umn . edu, hpfifer@umn. edu
}

measurements. These methods are based on the desired nominal performance [6]-[9], robust stability and/or robust performance [10]-[15]. Balas et al. [15] proposed a systematic approach for sensor selection based on $\mathcal{H}_{2}$ optimal control design techniques. This approach compares the performance objectives achieved including system constraints and is applied to an experimental structure. Dhingra et al. [8] considered the problem of sensor selection by evaluating the degradation of performance relative to a linear quadratic regulator that uses all available sensing capabilities. Lee et al. [13] developed screening tools to eliminate undesirable input/output candidates for which a robust controller does not exist. The screening tools are based on the structured singular value theory to compare the level of performance achieved in the presence of model uncertainty.

This paper extends the approach presented in [15] for sensor selection via closed-loop objectives by explicitly considering uncertainty in the aeroservoelastic system and synthesizing $\mathcal{H}_{\infty}$ robust controllers. The proposed method is applied to a small flexible aircraft with 8 control surfaces and 13 sensors available for feedback control. The goal is to find, out of a finite set of candidates, the best configuration of actuator/sensors that provides sufficient robustness to uncertainty in the input/output channels and achieves the performance objectives.

\section{PROBLEM FORMULATION}

For a given number of actuators and sensors available in the system for feedback control, we are interested in obtaining an optimal subset of control inputs and measurements that achieves the desired performance objectives and provides sufficient robustness to the model uncertainty.

The approach proposed to address this problem consists of two procedures. First, robust full-information (FI) controllers are designed for different actuator configurations, and their performance is compared to obtain an optimal selection of actuators. Second, robust full-control (FC) controllers are designed for different sensor configurations. Again, the performance is compared to optimally select sensors. Note that the full set of all combinatorial choices of actuators and sensors is large and unwieldly. Hence, this procedure is executed for a limited number of configurations to expedite the analysis.

The FI and FC problems are special cases of the output feedback control problem, where the FI plant provides the controller directly with states as measurements, while the FC plant assumes that the controller directly affects the states. The advantage of using the FI and FC formulations is that 
they are both convex problems. Hence, a globally optimal controller can be synthesized for each sensor/actuator configuration, and the controller performance for each configuration can be directly compared.

Other design techniques using output feedback control, without the FI and FC assumptions (e.g. $D K$-iteration [17], [18]), yield non-convex problems [19]. Because the $D K$ iteration does not synthesize globally nor locally optimal controllers, their performance does not only depend on the selected configuration but also the initial condition of the algorithm. Hence, their performance cannot be directly compared for different configurations. Thus, we use the FI and FC assumptions for this analysis.

A review of the mathematical details of the robust control formulation follows. Consider the general control synthesis configuration for uncertain systems in Fig. 1.

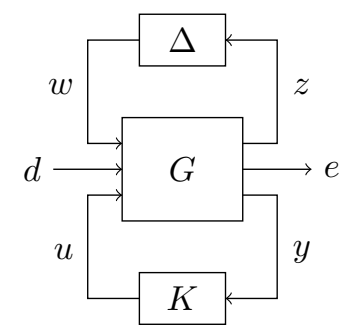

Fig. 1. Robust control configuration

Here $G$ is a linear time invariant (LTI) system, $K$ is the controller, and $\Delta$ is the model uncertainty. The performance objectives are collected in $e$, and the disturbances are collected in $d$. The controller is provided with measurements $y$ and generates control inputs $u$. In addition, the model uncertainty $\Delta$ is assumed to lie in the set

$$
\boldsymbol{\Delta}_{1 / \gamma}=:\left\{\operatorname{diag}\left(\Delta_{1}, \ldots, \Delta_{s}\right) \mid\left\|\Delta_{k}\right\| \leq \gamma^{-1}, k=1, \ldots, s\right\}
$$

The robust control problem is to find a stabilizing controller $K$ that minimizes the $\mathcal{H}_{\infty}$ norm of the closed-loop transfer function from $d$ to $e$ while maximizing the robustness against the uncertainty $\Delta$. This robust performance synthesis is defined by

$$
\inf _{K}\left(\sup _{\Delta \in \Delta_{1 / \gamma}}\left\|F_{U}\left(F_{L}(G, K), \Delta\right)\right\|\right) \leq \gamma
$$

where the linear fractional transformation $F_{U}\left(F_{L}(G, K), \Delta\right)$ denotes the uncertain closed-loop transfer function, $\|\cdot\|$ denotes the induced $L_{2}$ norm of the system, and $\gamma$ denotes the robust performance (RP) level achieved by the controller.

The optimization problem in (2) can be turned into a norm computation of a scaled system such that

$$
\inf _{K}\left(\inf _{\Theta \in \Theta}\left\|\Theta^{1 / 2} F_{L}(G, K) \Theta^{-1 / 2}\right\|\right)<\gamma
$$

where the scalings $\Theta$ belong to the set

$$
\Theta:=\left\{\operatorname{diag}\left(\theta_{1} I_{m_{1}}, \ldots, \theta_{s} I_{m_{s}}\right) \mid \theta_{k}>0, k=1, \ldots, s\right\}
$$

with $m_{k}$ as the dimension of the $k^{\text {th }}$ uncertainty block in (1). Note that with this definition each $\Theta \in \Theta$ has the property that it commutes with each $\Delta \in \Delta_{1 / \gamma}$, i.e. $\Theta \Delta=\Delta \Theta$.

The optimization problem in (3) is non-convex and there is no direct method to synthesize an optimal output feedback controller. However, this problem results in a convex optimization if the FI and FC special assumptions are considered for the control design.

In this section we derive the conditions for the FI problem. The conditions for the FC problem follow similarly based on the dual problem where $G_{F C}=G_{F I}^{T}$. The FI nominal plant, $G_{F I}$, is defined by the state space model

$$
G_{F I}:\left[\begin{array}{c}
\dot{x} \\
e \\
y
\end{array}\right]=\left[\begin{array}{ccc}
A & B_{1} & B_{2} \\
C_{1} & D_{11} & D_{12} \\
I & 0 & 0
\end{array}\right]\left[\begin{array}{l}
x \\
d \\
u
\end{array}\right]
$$

The $\mathcal{H}_{\infty}$ robust FI problem is to find a stabilizing controller $u=K x$ such that

$$
\inf _{\Theta \in \Theta}\left\|\Theta^{1 / 2} F_{L}\left(G_{F I}, K\right) \Theta^{-1 / 2}\right\|<\gamma
$$

where the scaled closed-loop transfer matrices $\Theta^{1 / 2} F_{L}\left(G_{F I}, K\right) \Theta^{-1 / 2}$ yields the state space

$$
\left[\begin{array}{c|c}
A+B_{2} K & B_{1} \Theta^{-1 / 2} \\
\hline \Theta^{1 / 2}\left(C_{1}+D_{12} K\right) & \Theta^{1 / 2} D_{11} \Theta^{-1 / 2}
\end{array}\right]
$$

The $\mathcal{H}_{\infty}$ norm computation in (6) can be transferred into a linear matrix inequality (LMI) optimization described by the following lemma.

Lemma 1 [16]. Let the scaled closed-loop transfer function in (7) be a stable LTI system. Then $\left\|\Theta^{1 / 2} F_{L}\left(G_{F I}, K\right) \Theta^{-1 / 2}\right\|<\gamma$ if there exists a symmetric matrix $\Theta \in \Theta$ and a symmetric matrix $P>0$ such that

$$
\begin{aligned}
& {\left[\begin{array}{cc}
\left(A+B_{2} K\right)^{T} P+P\left(A+B_{2} K\right) & P B_{1} \Theta^{-1 / 2} \\
\Theta^{-1 / 2} B_{1}^{T} P & -\gamma^{2} I
\end{array}\right]+} \\
& {\left[\begin{array}{c}
\left(C_{1}+D_{12} K\right)^{T} \\
\Theta^{-1 / 2} D_{11}^{T}
\end{array}\right] \Theta\left[\begin{array}{ll}
\left(C_{1}+D_{12} K\right) & D_{11} \Theta^{-1 / 2}
\end{array}\right]<0}
\end{aligned}
$$

The bilinear terms in (8) are simplified using the Schurcomplement. The result is then pre- and postmultiplied by the matrix $\operatorname{diag}\left(I, \Theta^{1 / 2}, \Theta^{-1 / 2}\right)$ to obtain

$$
\left[\begin{array}{ccc}
A^{T} P+P A+K^{T} B_{2}^{T} P+P B_{2} K & P B_{1} & C_{1}^{T}+K^{T} D_{12}^{T} \\
B_{1}^{T} P & -\gamma^{2} \Theta & D_{11}^{T} \\
C_{1}+D_{12} K & D_{11} & -\Theta^{-1}
\end{array}\right]<0
$$

As a final simplification, the first row and column of (9) are pre- and postmultiplied by $P^{-1}$. By defining $Q=P^{-1}$, $L=K Q$ and applying a Schur Complement, we obtain

$$
\begin{aligned}
& {\left[\begin{array}{cc}
Q A^{T}+A Q+L^{T} B_{2}^{T}+B_{2} L & Q C_{1}^{T}+L^{T} D_{12}^{T} \\
C_{1} Q+D_{12} L & -\Theta^{-1}
\end{array}\right]} \\
& +\frac{1}{\gamma^{2}}\left[\begin{array}{cc}
B_{1} \Theta^{-1} B_{1}^{T} & B_{1} \Theta^{-1} D_{11}^{T} \\
D_{11} \Theta^{-1} B_{1}^{T} & D_{11} \Theta^{-1} D_{11}^{T}
\end{array}\right]<0
\end{aligned}
$$

The LMI formulation in (10) is turned into a generalized eigenvalue problem to solve for a controller $K$ that minimizes the RP level $\gamma$. This eigenvalue problem is computed using existing software such as LMILab and YALMIP. 


\section{AEROSERVOELASTIC SYSTEM: BODY FREEDOM FLUTTER AIRCRAFT}

The Air Force Research Laboratory (AFRL) contracted with Lockheed Martin Aeronautics Company to develop a flight test vehicle, denoted Body Freedom Flutter (BFF) vehicle for demonstration of active aeroelastic control technologies [3], [20]. The vehicle is a high aspect ratio flying wing with light weight airfoil. The aircraft configuration with location of sensors and control surfaces is presented in Fig. 2. The aircraft has 8 control surfaces and 11 sensors available for control. Sensor measurements include gyros, accelerometers and hot-film sensors located at the leadingedge stagnation point (LESP) to estimate the lift distribution.

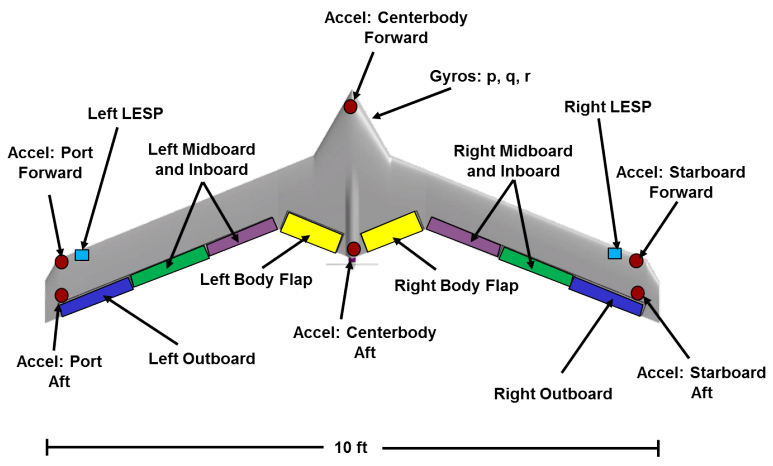

Fig. 2. Body Freedom Flutter Vehicle [3], [20]

Linear, continuous-time, state-space models of the airframe are generated at constant altitude of $1000 \mathrm{ft}$ from 40 to 90 KEAS (knots equivalent airspeed) with increments of 2 knots. Reduced order models with 17 states [21], [22], describe the aeroservoelastic behavior of the aircraft where three dynamic instabilities (flutter) are observed between $10-120 \mathrm{rad} / \mathrm{s}$ starting at 44,60 , and $62 \mathrm{KEAS}$. The control objective is flutter suppression and structural attenuation of flexible modes. Selection of appropriate surface and sensor measurements for feedback control is required to achieve the desired performance.

\section{A. Control surface selection}

The FI problem is used for selecting the appropriate set of actuation for the BFF aircraft. The interconnection used to synthesize the FI controllers is depicted in Fig. 3.

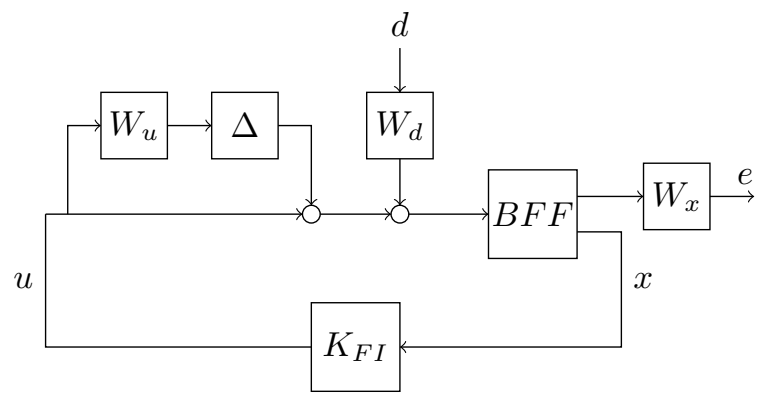

Fig. 3. Control interconnection for actuator selection
A constant performance weight $W_{x}$ is used to enforce damping to critical modes in the control bandwidth [23]. Three flexible modes corresponding to the symmetric wing bending, symmetric wing torsion and anti-symmetric wing torsion are selected as control objectives, with $W_{x}=8$ for all three modes.

Constant weights $W_{d}$ are used to model the disturbances to each control surface. This weight is selected as $W_{d}=0.5$ for all control surfaces. In addition, multiplicative input uncertainty is included to avoid destabilizing unmodeled high-frequency flexible modes outside the control bandwidth. The uncertainty weight represents approximately $40 \%$ model error up to $100 \mathrm{rad} / \mathrm{s}$ and as much as $100 \%$ uncertainty at $300 \mathrm{rad} / \mathrm{s}$ for each input channel. The uncertainty is represented by the weight, $W_{u}=500(s+121.4) /\left(s+1.6 \times 10^{5}\right)$. The uncertainty block $\Delta$ is diagonal with $\left\|\Delta_{k}\right\| \leq 1$ representing independent variations for each input channel.

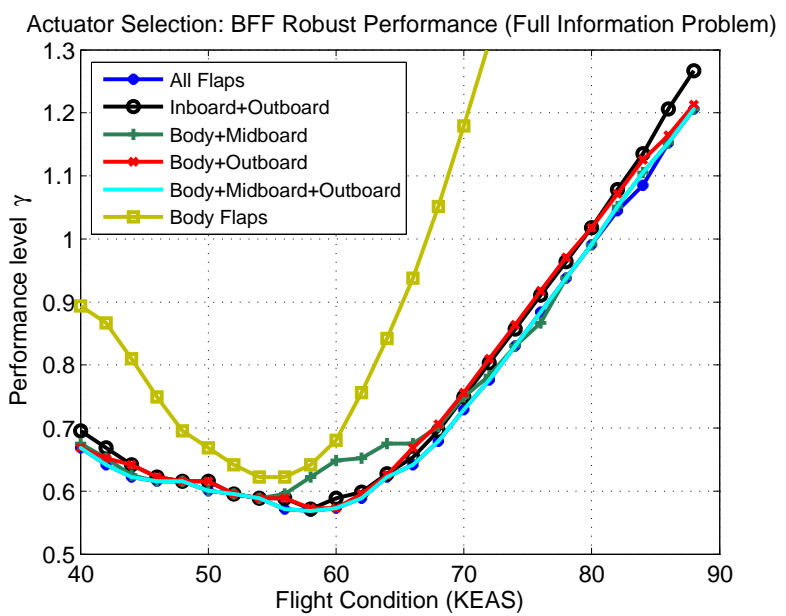

Fig. 4. $\gamma$-value variation across the flight envelope: actuator selection

FI controllers are synthesized by minimizing the RP level $\gamma$. It is noticed that stabilization of the flutter modes is the driving constraint in this control problem. Fig. 4 shows the variation of the $\gamma$-value across the flight envelope. It is observed that the different subsets of evaluated control surfaces do not have a significant effect in the performance of the aircraft unless only a pair of flaps is used. The combination that uses the Body and Outboard flaps shows a different result between 58 and 66 KEAS. Recall that the aircraft has 1 unstable mode at $58 \mathrm{KEAS}, 2$ unstable modes at 60 KEAS and 3 unstable modes beyond 62 KEAS. Notice that for all cases the performance starts to rapidly degrade when the aircraft has three unstable modes (62 KEAS).

Fig. 5 shows the phase disk margins obtained for each of the actuators used for control. A disk margin is the largest region for each channel that, for all simultaneous gain and phase variations inside the region, the nominal closed-loop system is stable. It is observed that the phase margins for the outboard flap are the most affected when the speed increases, even if all the available actuators are used. Using only body flaps results in, as expected, small 

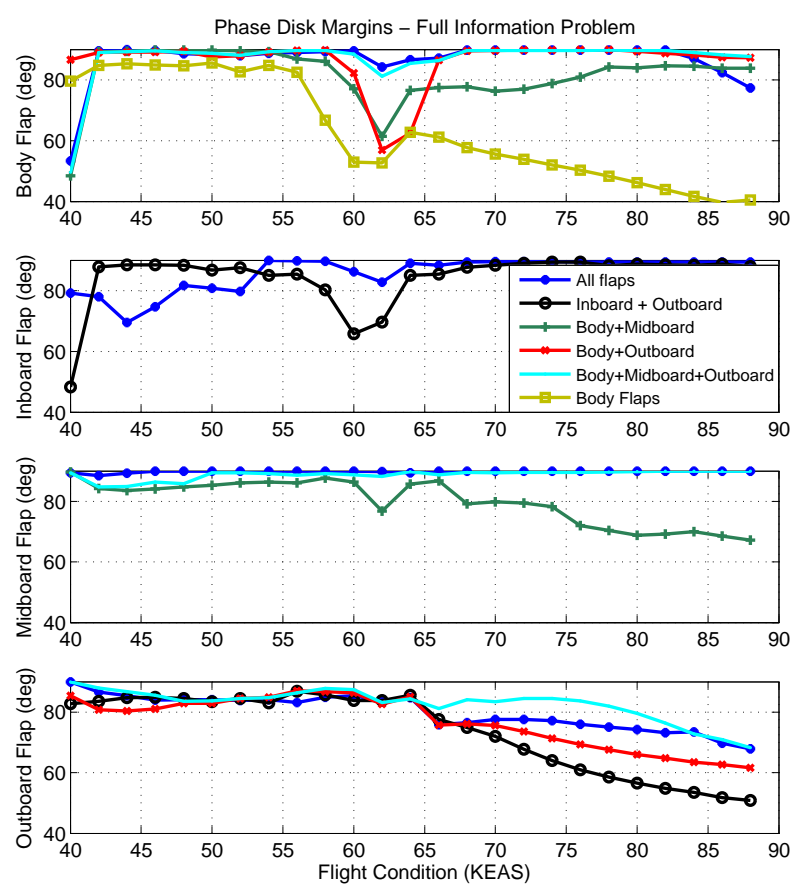

Fig. 5. Phase disk margins (loop-at-a-time): actuator selection

robustness margins when the aircraft has more than one unstable mode. On the other hand, the combination of three flap pairs (body+midboard+outboard) seems to obtain the best phase margins across the flight envelope. Gain margins are sufficiently large for all the combinations across the flight envelope which means that they do not constitute a constraint for actuator selection and thus, plots are not shown.

From this analysis, the conclusion is that the best combination of actuators to achieve the desired performance is the body, midboard and outboard flaps. In addition, the best combination of two pairs of surfaces seems to be the set of body and outboard flaps. Fig. 6 shows the closed-loop frequency response at $70 \mathrm{KEAS}$, for two optimal controllers synthesized using different control effectors. It is observed that the controller synthesized with two pairs of flaps successfully adds damping to the three flutter in contrast to the controller that only uses one pair of flaps.

\section{B. Sensor measurement selection}

The FC problem is used for selecting the appropriate set of measurements for the BFF aircraft. The interconnection used to synthesize the FC controllers is depicted in Fig. 7. Here, the flutter modes are directly affected by the input weight $W_{x}$. The control objective is the same as in the FI problem. The weights used are $W_{x}=1$ for all three modes and $W_{e}=1$ for all measurements.

Multiplicative output uncertainty is included to avoid sensitivity to measurement noise outside the control bandwidth. The uncertainty weight, $W_{u}=500(s+109.1) /(s+$ $\left.1.4 \times 10^{5}\right)$, represents approximately $40 \%$ model error up to $80 \mathrm{rad} / \mathrm{s}$ and as much as $100 \%$ uncertainty for frequencies higher than $300 \mathrm{rad} / \mathrm{s}$. Here, the diagonal uncertainty block

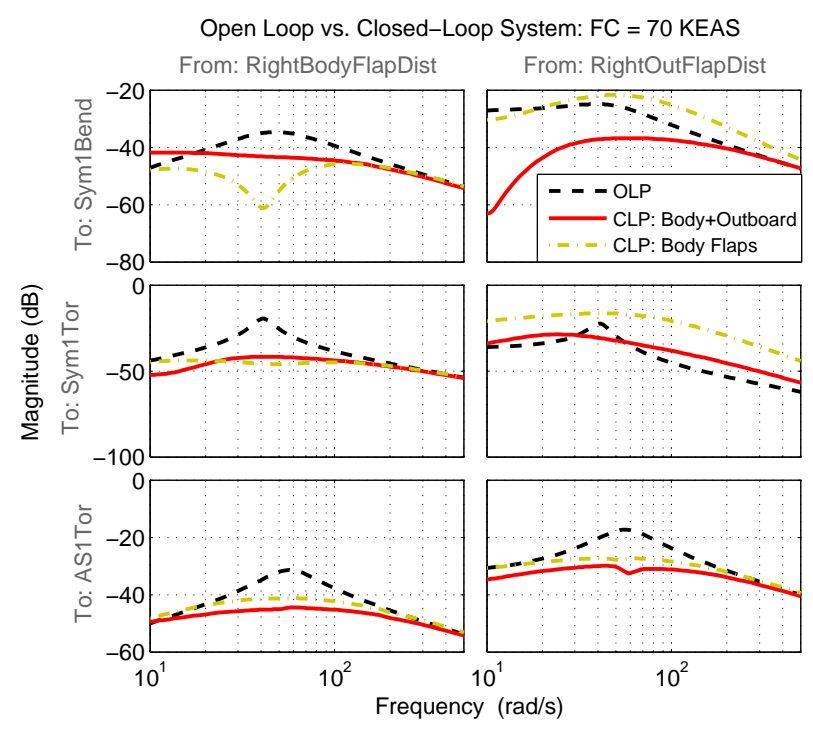

Fig. 6. Frequency response of damped closed-loop system at 70 KEAS: actuator selection

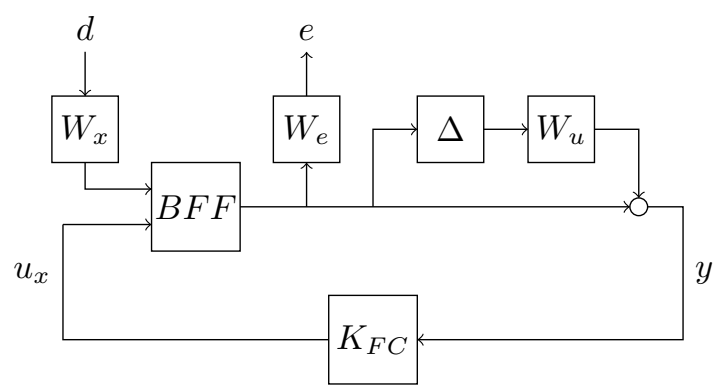

Fig. 7. Control interconnection for sensor selection

$\Delta$ with $\|\Delta\|_{k} \leq 1$ represents the sensor noise variation for each measurement.

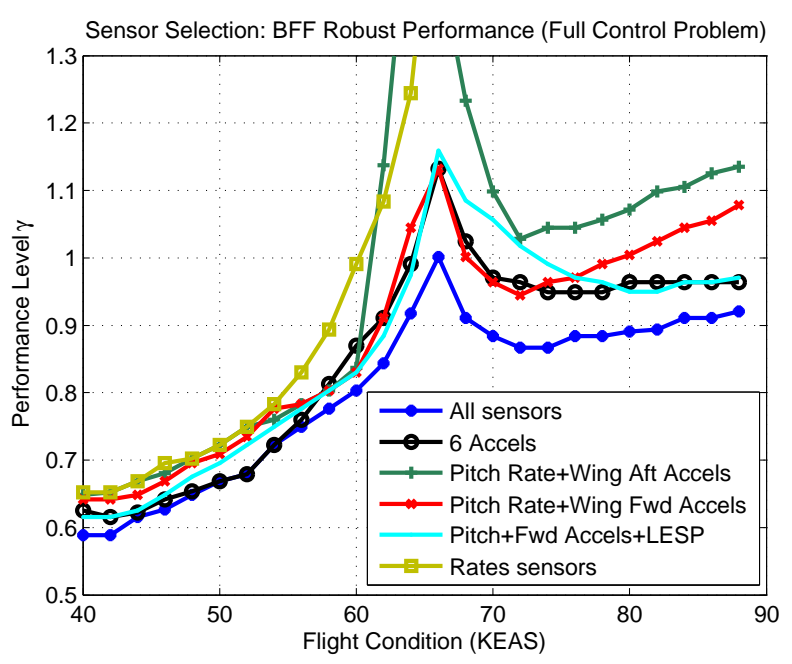

Fig. 8. $\gamma$-value variation across the flight envelope: sensor selection

Several sensor combination to synthesize FC controllers 
were tested but only the most relevant results are plotted. Fig. 8 shows the variation of RP $\gamma$-level across the flight envelope for six different sets of measurements. It is observed that the robust performance of the system is more sensitive to the selection of sensors than to the selection of actuators as shown in the previous subsection.

The best robust performance is given, as expected, by the usage of all 11 available measurements. Notice that using all the acceleration information of the aircraft, which corresponds to 6 accelerometers distributed between the body and both wings, obtains acceptable robust performance. It is observed that for all cases, the performance is degraded across the flight envelope and it is critical at 66 KEAS. At this critical speed, the body freedom flutter is close in proximity to the symmetric torsional flutter mode.

In addition, a comparison between the set of sensors corresponding to the pitch rate together with the wing aft/forward accelerometers is shown in Fig. 8. Here, the combination that includes the accelerometers at the leading edge of the wing provide better information to the controller than the accelerometers at the trailing edge. It is also noticed that the performance for the leading edge set, compared to the set with 6 accelerometers, is degraded for flight conditions starting at $74 \mathrm{KEAS}$. In this case, the body freedom flutter is close in proximity to the anti-symmetric torsional flutter mode. This means that acceleration information from both leading and trailing edge is required to successfully damp out the anti-symmetric mode. In addition, using the LESP sensors together with the pitch rate and leading edge accelerometers can improve the performance at high speeds.

On the other hand, it is observed that using only the vehicle rates for feedback, the performance is significantly degraded across the flight envelope, starting at $58 \mathrm{KEAS}$ when the aircraft is in transition to have two flutter modes.

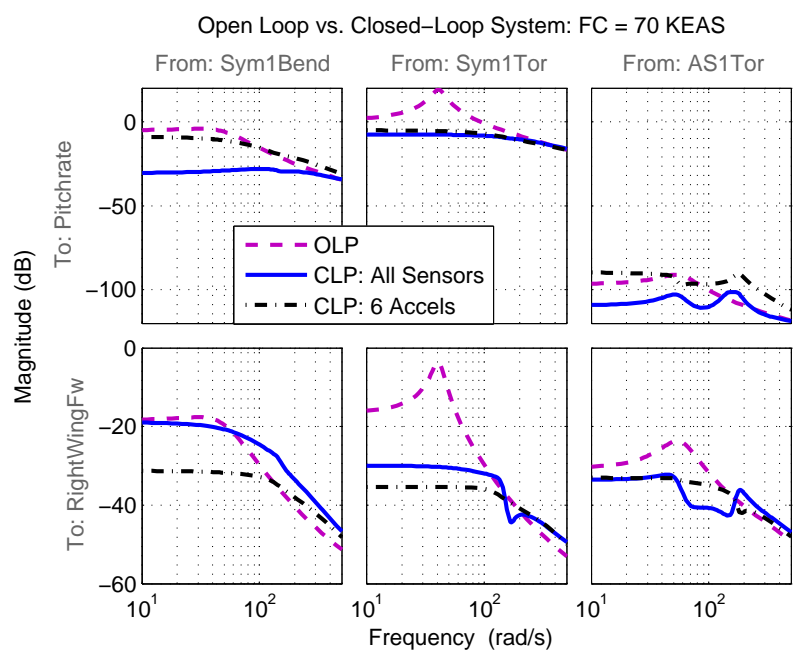

Fig. 9. Frequency response of damped closed-loop system at 70 KEAS: sensor selection

Fig. 9 compares the closed-loop system damping for two sensor combinations at $70 \mathrm{KEAS}$. It is observed in the plot that the controller synthesized with all available sensors can provide more damping to the anti-symmetric flutter mode than the combination with all 6 accelerometers.

Fig. 10 shows the phase disk margins obtained for each of the measurements used for control. It is observed that the phase margins obtained with the controller using all 6 accelerometers for feedback, are acceptable and in some cases better than the margins provided when all available sensors are used. Gain margins, as in the actuator selection case, are sufficiently large for all the combinations across the flight envelope.

From this analysis, the conclusion is that the best combination of sensors for control feedback, given the desired performance and robustness, is achieved by using the 6 accelerometers available in the aircraft.

\section{CONCLUSIONS}

An approach for selection of actuators and sensors for aeroservoelastic systems is described in this paper. The approach is based on robust controller synthesis for the special cases of the FI and FC problem. The FI and FC problem provide a globally optimal controller that allows a fair comparison between different sensor/actuator combinations.

The proposed actuator/sensor selection method was applied to a particular aeroservoelastic aircraft with three flutter modes across the flight envelope. From this analysis it is concluded that the best subset of control surfaces corresponds to the body and outboard flaps. Following, the best subset of sensor measurements for feedback corresponds to the combination with all the accelerometers available in the aircraft. Output feedback controllers will be designed using the set of actuator and sensor measurements obtained from this procedure. Notice that the performance obtained by output feedback controllers can only be expected to be worse than the performance levels reported.

Future work will incorporate a sparsity condition for the controller into the optimization problem. The goal is to minimize the number of nonzero rows or columns in order to find the minimum subset of actuator/sensors that will achieve the desired robust performance.

\section{ACKNOWLEDGMENT}

This research is supported by the NASA STTR contract No. NNXIICI09P entitled Robust Aeroservoelastic Control Utilizing Physics-Based Aerodynamic Sensing as a subcontract from Tao Systems. Dr. Arun Mangalam is the principal investigator and Dr. Martin Brenner is the NASA technical monitor. This work was also partially supported by NASA under Grant No. NRA NNX12AM55A entitled Analytical Validation Tools for Safety Critical Systems Under Loss-ofControl Conditions, Dr. C. Belcastro is the technical monitor.

The first author gratefully acknowledges the financial support from University of Minnesota through the 2014 Doctoral Dissertation Fellowship. 

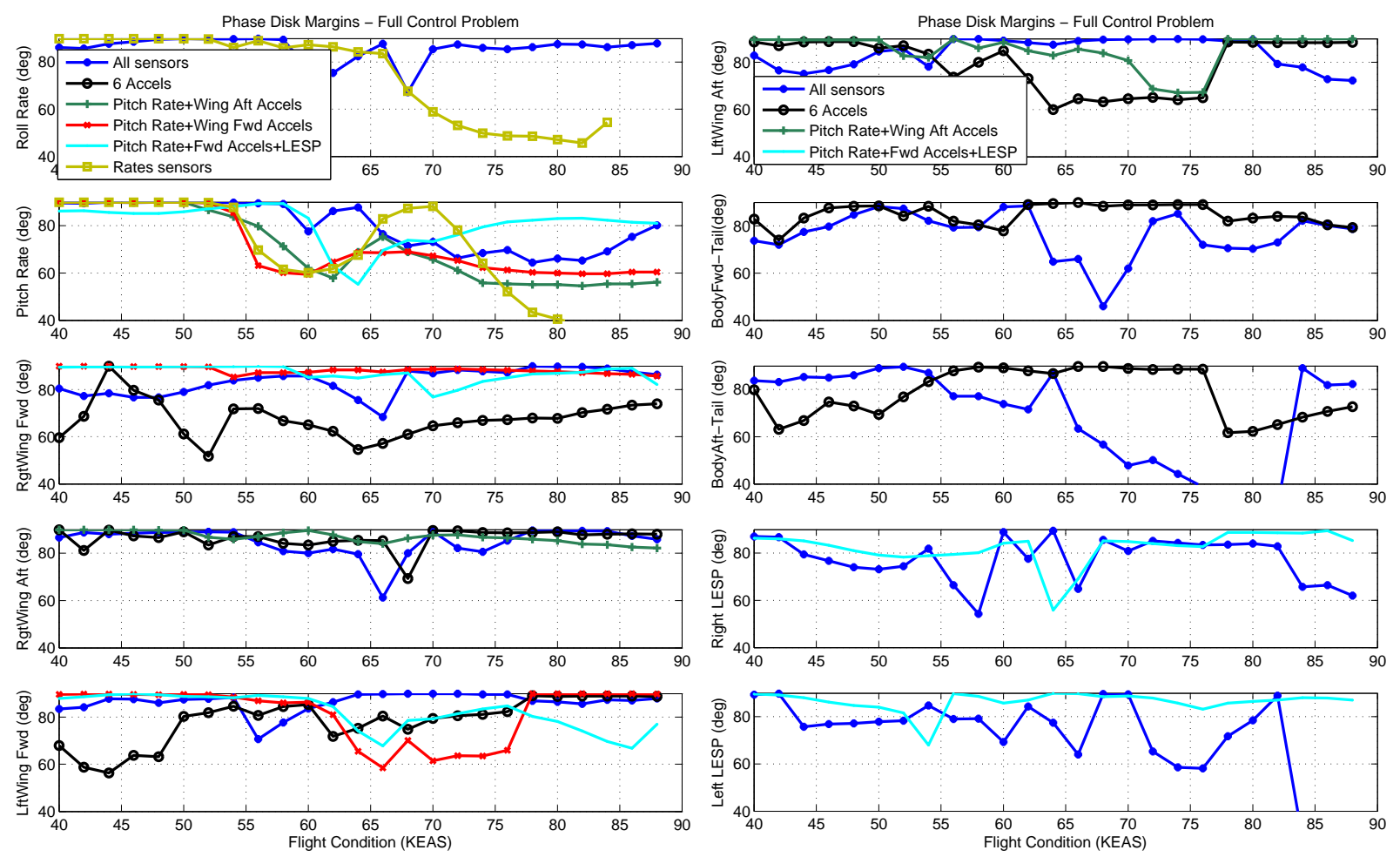

Fig. 10. Phase disk margins (loop-at-a-time): sensor selection

\section{REFERENCES}

[1] Waszak, M., "Robust multivariable flutter suppression for the benchmark active control technology (BACT) wind-tunnel model," 11th Symposium on Struct. Dynamics and Control, Blacksburg, VA, 1997.

[2] Van Etten, C., Balas, G. J., and Bennani, S., "Linear parameter-varying integrated flight and structural mode control for a flexible aircraft," AIAA Guidance, Navigation and Control Conf., Portland, OR, 1999.

[3] Holm-Hansen, B., Atkinson, C., Benarek, J., Burnett, E., Nicolai, L., and Youssef, H., "Envelope expansion of a flexible flying wing by active flutter suppression," AUVSI's Unmanned Systems North America, Denver, CO, 2010.

[4] Balas, G. J., Moreno, C. P., and Seiler, P., "Robust Aeroservoelastic Control Utilizing Physics-Based Aerodynamic Sensing," AIAA Atmospheric Flight Mechanics Conference, Minneapolis, 2012.

[5] Skogestad, S., Morari, M., and Doyle, J. C., "Robust control of ill-conditioned plants: high purity distillation," IEEE Transactions Automatic Control, Vol. 33, 1988, pp. 1092-1105.

[6] Weber, R. and Brosilow, C. B., "Use of secondary measurements to improve control," AIChE Journal, Vol. 18, 1972, pp. 614-623.

[7] Joseph, B. and Brosilow, C. B., "Inferential control of processes," AIChE Journal, Vol. 24, 1978, pp. 910-916.

[8] Dhingra, N. K., Jovanovic, M. R., and Luo, Z. Q., "Optimal sensor and actuator selection for large-scale systems," in Proceedings of the 53rd IEEE Conf. on Decision and Control, Los Angeles, CA, 2014.

[9] Albertos, A., "Relevance of actions and measurements in control performance," Australian Journal of Electrical and Electronics Engineering, Vol. 2, No. 2, 2005, pp. 159-166.

[10] Van de Wal, M., Philips, P., and De Jager, B., "Actuator and sensor selection for an active vehicle suspension aimed at robust performance," International Journal of Control, Vol. 70, No. 5, 1998, pp. 703-720.

[11] Rivera, D. E., "A structural singular value test for variable selection and pairing," Proceedings of 1989 American Control Conference, Pittsburgh, PA, pp. 560-565.

[12] Braatz, R. D., Lee, J. H., and Morari, M., "Screening plant designs and control structures for uncertain systems," Computers and Chemical Engineering, Vol. 20, 1996, pp. 463-468.

[13] Lee, J. H., Braatz, R. D, Morari, M., and Packard, A., "Screening tools for robust control structure selection,"Automatica, Vol. 31, No. 1, 1995, pp. 229-235.
[14] van de Wal, M. and de Jager, Bram, "A review of methods for input/output selection", Automatica, Vol. 37, pp. 487-510.

[15] Balas, G. J. and Young, P. M., "Sensor selection via closed-loop control objectives," IEEE Transactions on Control Systems Technology, Vol.7(6), 1999, pp.692-705.

[16] Dullerud, G. and Paganini, F., A course in robust control theory: A convex approach, Text in Applied Mathematics, No. 36, Springer, New York, NY, 2000.

[17] Balas,G., Doyle, J., Glover, K., Packard, A., and Smith, R., " $\mu$ Analysis and synthesis toolbox: Users guide," MUSYN and The Mathworks, Natick, MA, June 2001.

[18] Packard, A., Doyle, J., and Balas, G., "Linear multivariable robust control with a $\mu$ perspective," Journal of Dynamics, Measurements and Control: Special Edition on Control, Vol. 115, No. 2(B), 1993, pp. 426-438.

[19] Stein, G. and Doyle, J., "Beyond singular values and loop shapes," Journal of Guidance, Control, and Dynamics, Vol. 14, No. 1, 1991, pp. 5-16.

[20] Burnett E., Atkinson, C., Beranek, J., Sibbitt, B., Holm-Hansen, B. and Nicolai, L., "NDOF simulation model for flight control development with flight test correlation," AIAA Modeling and Simulation Technologies Conference, Vol. 3, Toronto, Canada, 2010, pp. 7780-7794.

[21] Moreno, C. P., Seiler, P., and Balas, G. J., "Model reduction for aeroservoelastic systems," Journal of Aircraft, Vol. 51, No. 1, January 2014.

[22] Moreno, C. P., Seiler, P., and Balas, G. J., "Linear, parameter varying model reduction for aeroservoelastic systems," AIAA Atmospheric Flight Mechanics Conference, Minneapolis, 2012.

[23] Theis, J., Pfifer, H., Balas, G. and Werner, H., "Integrated control design for a large flexible aircraft," American Control Conference, Chicago, IL, 2015. 